\title{
Futures Trend Strategy Model Based on Recurrent Neural Network
}

\author{
Ru Zhang ${ }^{1}$, Chenyu Huang ${ }^{2} \&$ Shaozhen Chen ${ }^{1}$ \\ ${ }^{1}$ Finance Department of International Bussiness School, Jinan University, Zhuhai, Guangdong Province, China \\ ${ }^{2}$ Financial Management Department of International Bussiness School, Jinan University, Zhuhai, Guangdong Province, \\ China \\ Correspondence: Shaozhen Chen, Finance Department of International Business School, Jinan University, Qianshan \\ Road 206\#, Zhuhai City, Guangdong Province, Post No. 519070, China.
}

Received: May 26, 2018

doi:10.11114/aef.v5i4.3306
Accepted: June 16, $2018 \quad$ Available online: June 19, 2018

URL: https://doi.org/10.11114/aef.v5i4.3306

\begin{abstract}
In recent years, quantitative investment has been widely used in the global futures market, and its steady investment performance has also been recognized by domestic futures investors. This paper takes the CSI-300 stock index futures as the research object and constructs a futures trend strategy model based on recurrent neural network. Furthermore, this paper back tests the strategy at different periods, different transaction costs and different parameters. The results show that the strategy model has strong profitability and robustness.
\end{abstract}

Keywords: futures trend strategy, recurrent neural network, quantitative trading

\section{Introduction}

Quantitative investment is defined as the combination of investment ideas or theories of investors with advanced computer technology and various disciplines, which were transformed into mathematical models. Then, specific investment decision analysis and implementation will be done by computer. In quantitative investment, trend strategy is one of the most common models. Shik et al. (2007) forecasted the role of indicators in the foreign exchange market through the statistics studies and analysis of two trend indicators: $R S I$ and $M A$. Elsevier et al (2009) proposed that trading methods based on historical price fluctuations and traditional technology analysis are able to bring more benefits. Yumin Zhou (2015) upgraded the returnee transaction method by upgrading filters and delisting and optimizing parameters. At the same time, he obtained a significant increase in back testing indicators in the testing of gold futures. Chen et al. (2016) used the LASSO method to select the smallest technical index as the judgment function, and used the genetic network plan to find the appropriate sale points. Then, he constructed the strategy so as to obtain more benefits. $\mathrm{Li}$ et al. (2017) studied the performance of buy-hold strategy and mobile average strategy in single variety futures and grouping combinations, and found that the income of mobile average technology is significantly higher than that of buy-hold strategy which had appears in China futures market. However, the traditional trend strategy still has weaknesses such as short and weak trend and non robust parameters, which can easily lead to transaction errors that would be caused by repeated transactions and model failure. Chi et al. (1991) found out that recurrent neural network would remember the previous information and apply it to the current calculation; therefore it has a good prediction effect on the price trend of stock index futures. Tenti et al. (2017) concluded artificial neural networks, including $R N N$, are efficient in the prediction of financial time series, and have carried out a comparative experiment on the prediction of foreign exchange prices.

To sum up, this paper takes the CSI-300 stock index futures as the research object. First, we classify the training data through the trend strategy profit index, study the parameter $R N N$ and then constructed the futures trend strategy based on recurrent neural network, which is tested by the minute market data for its profitability. Secondly, under different transaction costs and policy parameters, the strategy is compared to test its robustness. Throughout the entire research, this paper hopes to build a quantitative investment strategy in deep learning to provide further improvement for the application of artificial intelligence theory in the field of investment. 


\section{Theoretical Model}

\subsection{Recurrent Neural Network Model}

\subsubsection{Basic Principles of Recurrent Neural Network}

The recurrent neural network algorithm is very good at handling data that depend on timing. As shown in Figure 1, in the traditional forward neural network, the transmission of information from input layer to hidden layer, and to output layer, are fully connected. However, the nodes at different times are unconnected, and the network cannot deal with the timing problem directly. However, in recurrent neural network, there are connections between the nodes of the hidden layers at different time. The current output of a sequence is depended on the previous state, which means that the network will memorize the preceding information and apply it to the calculation of the current output.
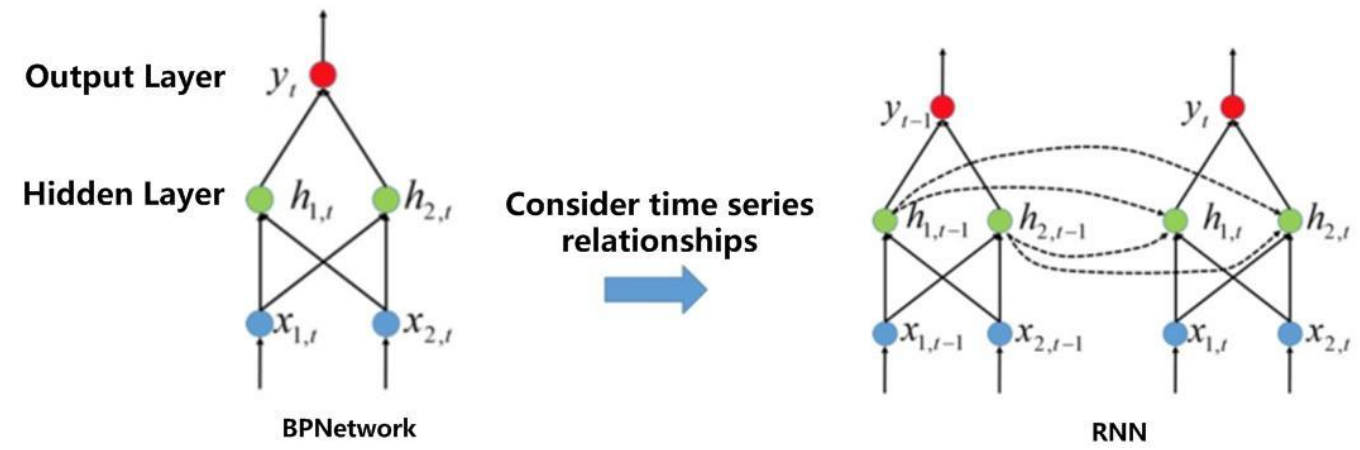

Figure 1. Recurrent neural network schematic

$R N N$ can be regarded as a neural network that shares weights on time dimension. The output of the $R N N$ model is as follows:

$$
y_{t}=\sigma_{0}\left(W_{y} h_{t}+b_{0}\right)
$$

The output of the model depends on the hidden state $h_{t}$ at that time and the hidden state $h_{t}$ depends on the input $x_{t}$ of the time and the hidden state $h_{t-1}$ at the previous moment, that is:

$$
h_{t}=\sigma_{h}\left(W_{x} x_{t}+W_{h} h_{t-1}+b_{n}\right)
$$

Therefore, the implicit state is time dependent, so the output of the $R N N$ model is related to the input information of the previous time.

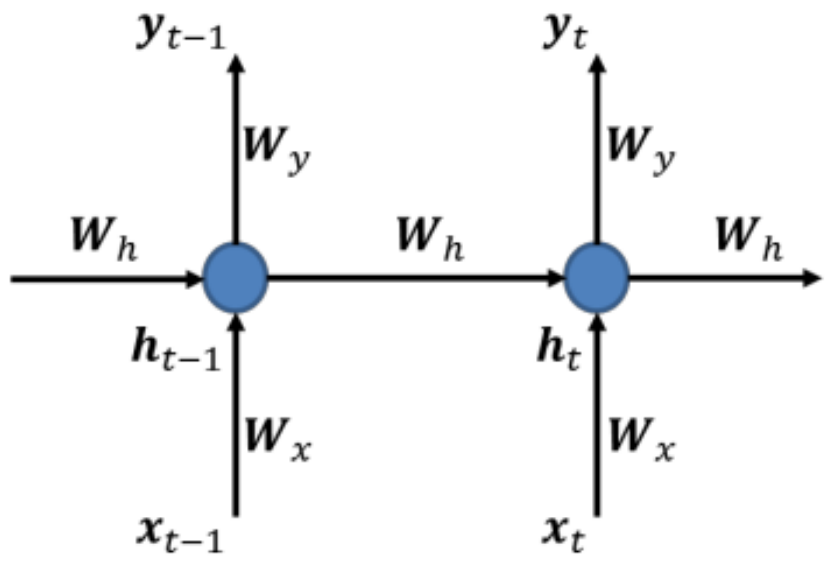

Figure 2. $R N N$ Parametric schematic diagram

The relationship between the RNN model and the ordinary time series model can be analyzed in the following way, which considers the single variable situation: $x_{t}=x_{t}, W_{h}=\beta, W_{x}=\alpha$. When output $y_{t}=h_{t}$, assuming that $\sigma_{h}$ is an identity transformation, the $R N N$ model can be transformed into the following forms:

$$
y_{t}=\alpha x_{t}+\beta y_{t-1}+b
$$

In essence, the model $R N N$ can be regarded as a first-order autoregressive model with exogenous variables, so it is a complex nonlinear time series model. 


\subsubsection{Parameter Optimization of RNN Model}

Gradient of the recurrent neural network can be obtained through Back Propagation Through Time (BPTT) Algorithms

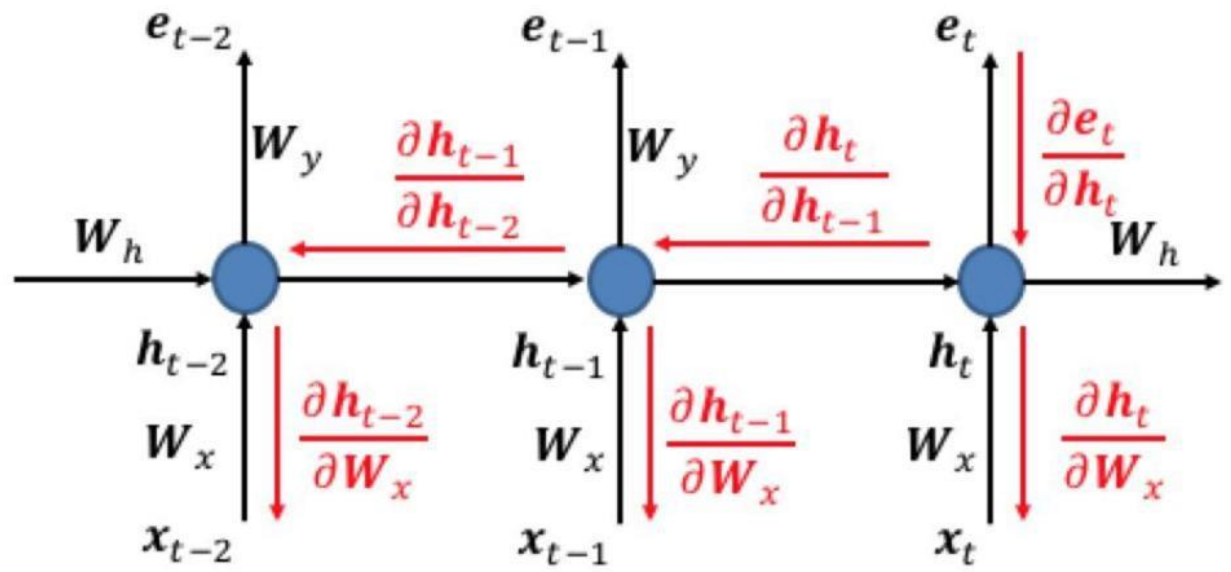

\section{BPTT Algorithm}

Figure 3. RNN parameter learning diagram

In the RNN model, since the implicit state $h_{t}$ of the RNN is affected by the implicit state $h_{t-1}$ at that time, the gradient of the RNN is related to the sequence. The BPTT is based on the sequence-dependent assumption of the RNN. The loss function is set at each sequence point, and the final loss function $\mathrm{L}$ is obtained as the gradient $\delta_{t}$ of the implicit state at each sequence point:

$$
\begin{aligned}
L & =\sum_{t=1}^{T} L_{t} \\
\delta_{t} & =\frac{\partial L}{\partial h_{t}}
\end{aligned}
$$

According to the final loss function, the gradient calculation formula of each parameter of RNN can be further deduced, which is:

$$
\begin{aligned}
& \frac{\partial L}{\partial c}=\sum_{t=1}^{T} \frac{\partial L_{t}}{\partial c}=\sum_{t=1}^{T} \frac{\partial L}{\partial \sigma_{t}} \frac{\partial \sigma_{t}}{\partial c}=\sum_{t=1}^{T} y_{t}-y_{t} \\
& \frac{\partial L}{\partial V}=\sum_{t=1}^{T} \frac{\partial L_{t}}{\partial V}=\sum_{t=1}^{T} \frac{\partial L}{\partial \sigma_{t}} \frac{\partial \sigma_{t}}{\partial V}=\sum_{t=1}^{T}\left(y_{t}-y_{t}\right)\left(h_{t}\right)^{T} \\
& \frac{\partial L}{\partial W}=\sum_{t=1}^{T} \frac{\partial L}{\partial h_{t}} \frac{\partial h_{t}}{\partial W}=\sum_{t=1}^{T} \operatorname{diag}\left(1-h_{t}^{2}\right) \delta_{t}\left(h_{t-1}\right)^{T} \\
& \frac{\partial L}{\partial b}=\sum_{t=1}^{T} \frac{\partial L}{\partial h_{t}} \frac{\partial h_{t}}{\partial b}=\sum_{t=1}^{T} \operatorname{diag}\left(1-h_{t}^{2}\right) \delta_{t} \\
& \frac{\partial L}{\partial U}=\sum_{t=1}^{T} \frac{\partial L}{\partial h_{t}} \frac{\partial h_{t}}{\partial U}=\sum_{t=1}^{T} \operatorname{diag}\left(1-h_{t}^{2}\right) \delta_{t}\left(x_{t}\right)^{T}
\end{aligned}
$$

\subsection{Trend Strategy Model}

This study used the following trend strategy profitability indicators as the original signal generator for trend judgment, expressed as:

$$
R=\frac{\mid \text { Close }- \text { Open } \mid}{\text { High }- \text { Low }}
$$

$\mathrm{R}$ represents the proportion of the entity part of the Candle line on the same day. In general, when $\mathrm{R}$ is relatively large, the trend strategy is easier to get profits; otherwise, the trend strategy is more difficult to be profitable. 


\subsection{Futures Trend Strategy Model Based on Recurrent Neural Network}

As can be seen in Figure 4, market data of the parameter T length is taken after the opening date of each trading day, and the RNN model is used to predict whether the trend strategy of the day can be profitable or not. If it is profitable, the trends in the early morning is followed for trend tracking purposes; if the model determines that it cannot make profits, no trading will be open on that day.

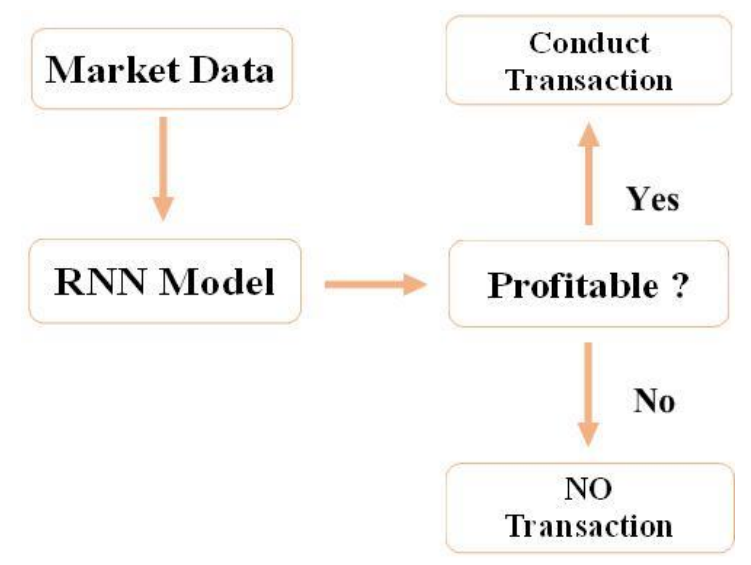

Figure 4. Schematic diagram of strategy

Before trading, the RNN prediction model training is required. This study adopted the "time series input - single label output" model structure. That is to say that the input data is a multivariate time series, and the output data is 0,1 tag data. In model training, early-morning quotes for the data in the sample need to be annotated, and the market is marked as two different categories of "suitable for trend transactions" and "not suitable for trend transactions" which means that the trend strategy indicator is calculated to determine whether the trend strategy of the day is profitable: when $R>0.5$, it is profitable, and recorded as "1" (for trend trading); when $R<0.5$, loss, and recorded as "0" (not suitable for trend trading). The labeled sample data is used to train machine learning models.

After the open trade, the position is held until the close of the day, and if a stop is triggered, the position will be closed immediately after the stop loss is triggered.

\section{Empirical Analysis}

\subsection{Data Selection and Description}

(1). One-minute price of the CSI-300 Stock Index Futures (IF) main contract. The data from April 16th, 2010 to December 31st, 2013 are sampled. The RNN forecasting model is trained using the market data for this period of time; while it is the out-of-sample return interval from January 1st, 2014 to July 31st, 2017.

(2) The characteristic data sequence is the minute-level market data of the opening price, the closing price, the highest price, the lowest price, the trading volume, the main buying amount, the main selling amount, the change rate of the closing price, and the closing price sequence of the minute market, order differential, ratio of main buyer sales, volume change rate, change rate of main purchase volume, change rate of main sales volume. Feature data is standardized.

\subsection{Transaction Parameter Settings}

(1). Back test margin setting: $100 \%$, ie no leverage is taken into consideration.

(2). Transaction costs: $100 \%$, ie no leverage is taken into consideration.

(3). Stop loss setting: stop loss according to a fixed ratio

\subsection{Model Evaluation Index}

This study selects the annualized interest rate, the cumulative yield, the maximum retracement, the winning ratio, the profit-loss ratio, and the single-transaction yield as indicators of the evaluation strategy.

\subsection{Simulation Back Testing}

This study selected 33-minutes after the opening to forecast trend strategy profit index $R$ of the full-day. The trend of the profitability probability P is shown in Figure 5, and the out-of-sample forecast accuracy rate is $59.1 \%$. 


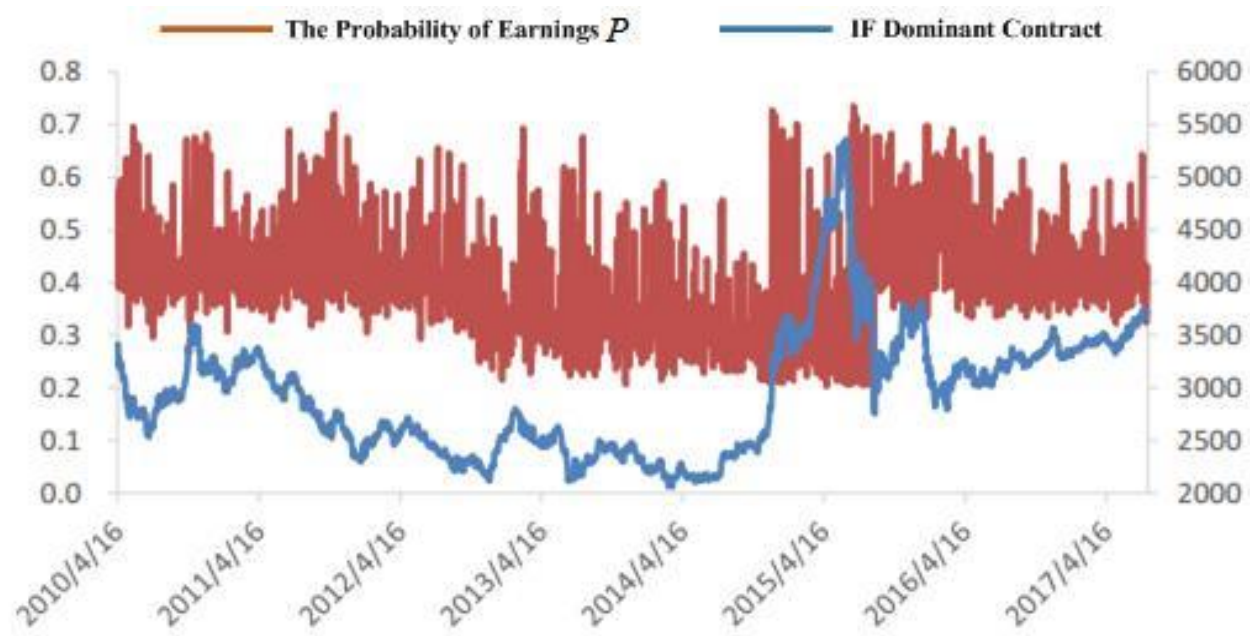

Figure 5. Profit Probability Chart

The performance of the strategy model across the entire sample interval is shown in Figure 6. Since April 2010, the strategy has been traded a total of 707 times, achieving a cumulative yield of $223.46 \%$, an annualized rate of return of $18.01 \%$, a maximum retracement of $-8.63 \%$, and a profit-to-loss ratio of 2.17 .

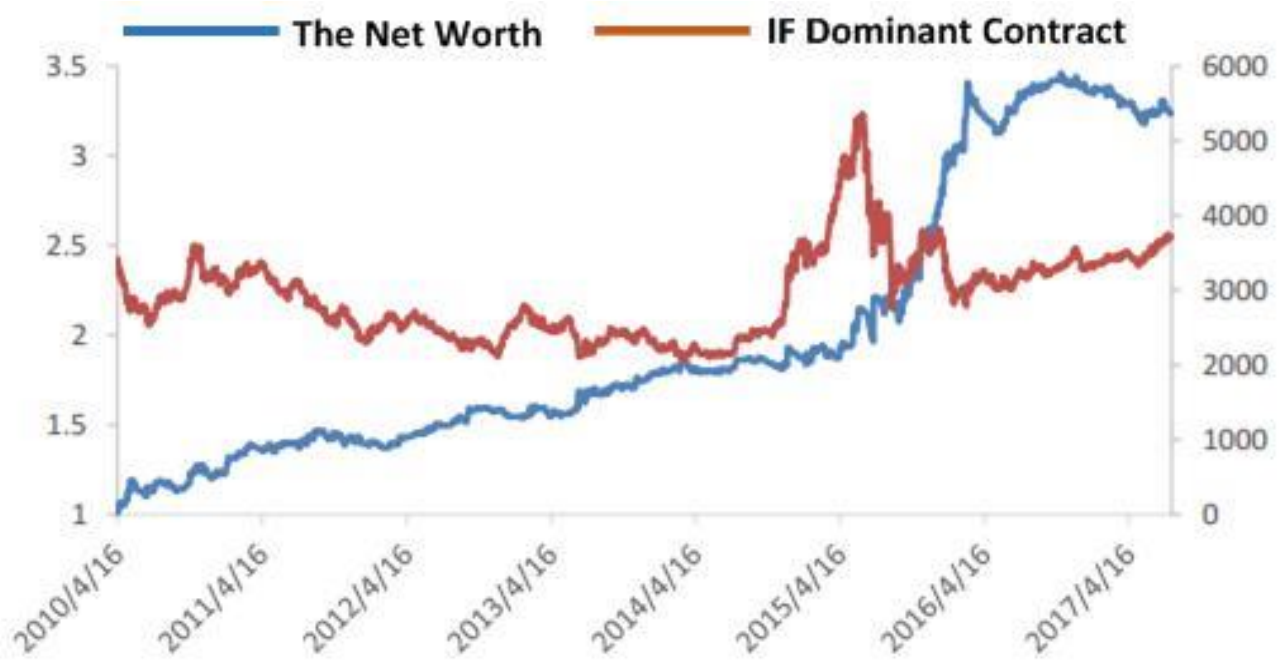

Figure 6. Full sample performance

Table 1. Strategy Performance in 2010

\begin{tabular}{cc}
\hline Index & Value \\
\hline Time yield & $18.01 \%$ \\
Cumulative return & $223.46 \%$ \\
Maximum retracement & $-8.63 \%$ \\
Transaction number & 707 \\
odds & $40.74 \%$ \\
Profit and loss than & 2.17 \\
Yield per trade & $0.17 \%$ \\
\hline
\end{tabular}

This strategy model is a trend trading strategy with a stop loss mechanism, so the winning rate is not high, which is $39.52 \%$, but the profit and loss of the strategy is high at $2.27 \%$. Strategy of a single transaction is an average yield of $0.17 \%$. Compared with other indexes of CTA short term strategy, the strategy of a single transaction average yield is higher. Therefore, the sensitivity to transaction costs and market impact costs is low. Since September 2015, stock index futures trading have been greatly restricted, transaction costs increase, market liquidity becomes worse and impact costs increase. Therefore, the average return rate of a single transaction of CTA strategy is an important index. If the transaction costs raise to bilateral five over ten thousand, the performances of the strategy outside the sample are shown in figure 7. In the case that transaction costs sharply raised, the performance of the strategy is slightly lower. However, since 2014 , the annual yield of the strategy is $14.71 \%$ and a single trading average yield is $0.14 \%$. Generally, the 
performance is still good, which means that this transaction strategy is not sensitive to transaction costs.

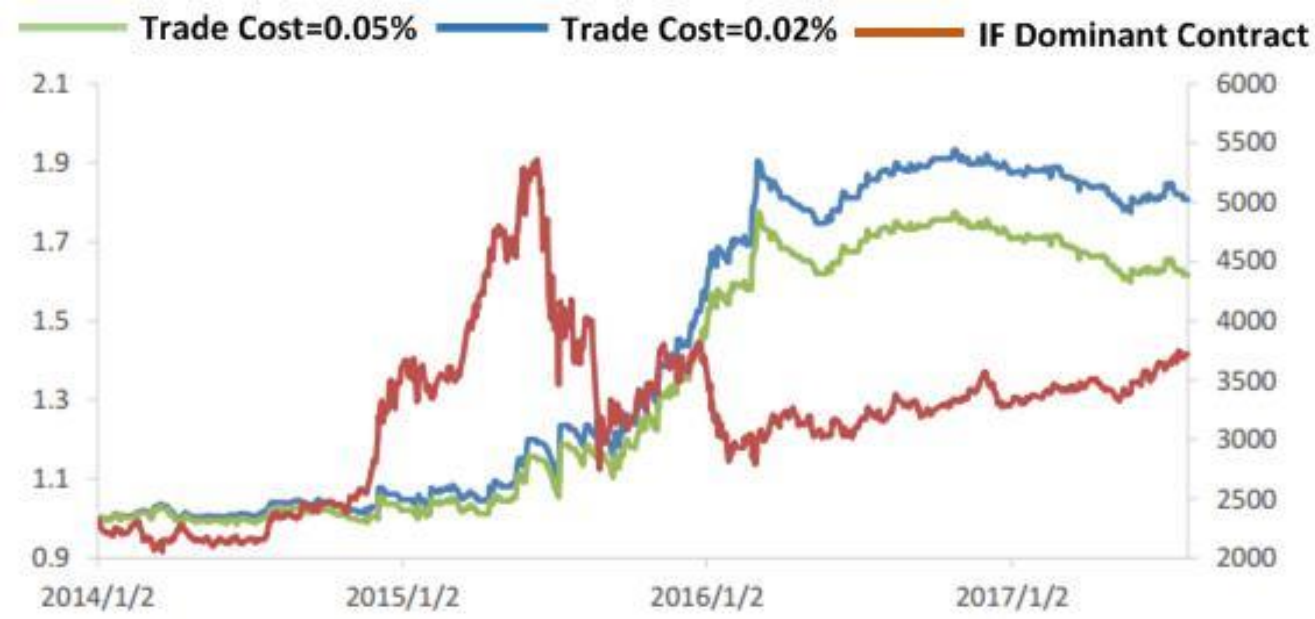

Figure 7. strategic net worth at different transaction costs (out of sample)

Table 2. Strategic performance in 2014

\begin{tabular}{cc}
\hline Index & Value \\
\hline Annual return & $18.47 \%$ \\
Cumulative rate of return & $80.72 \%$ \\
Maximum withdrawal & $-8.63 \%$ \\
Number of transactions & 372 \\
Winning rate & $39.52 \%$ \\
Profit and loss ratio & 2.27 \\
Single transaction yield & $0.17 \%$ \\
\hline
\end{tabular}

\subsection{Parameter Sensitivity Analysis}

Profit and loss ratio

The opening time $\mathrm{T}$ is the main parameter of this strategy, and $T=33$ minute is the optimal opening time parameter obtained from the strategy in this report. In order to analyze the sensitivity of parameters, different $T$ were selected for back measurement. The specific strategy is the same as above; the daily trend strategy profit probability $P$ is predicted based on the market data after the $T$ opening minutes, and then the daily 120 average daily of the probability $P$ at $T$ time is calculated. If $P>M A_{120}(P)$, then trend trading is carried out. According to the main contract of IF after the opening $T$ minutes, the trend direction is established from the direction of fluctuation; Otherwise, do not open a position on the day trading.

Under different parameter settings, the performance of the strategy outside the sample is shown in figure 8 . Any time strategy that is performed during 22 minutes to 38 minutes after opening to trade, it shows good performance; therefore it explains that this strategy has good stability.

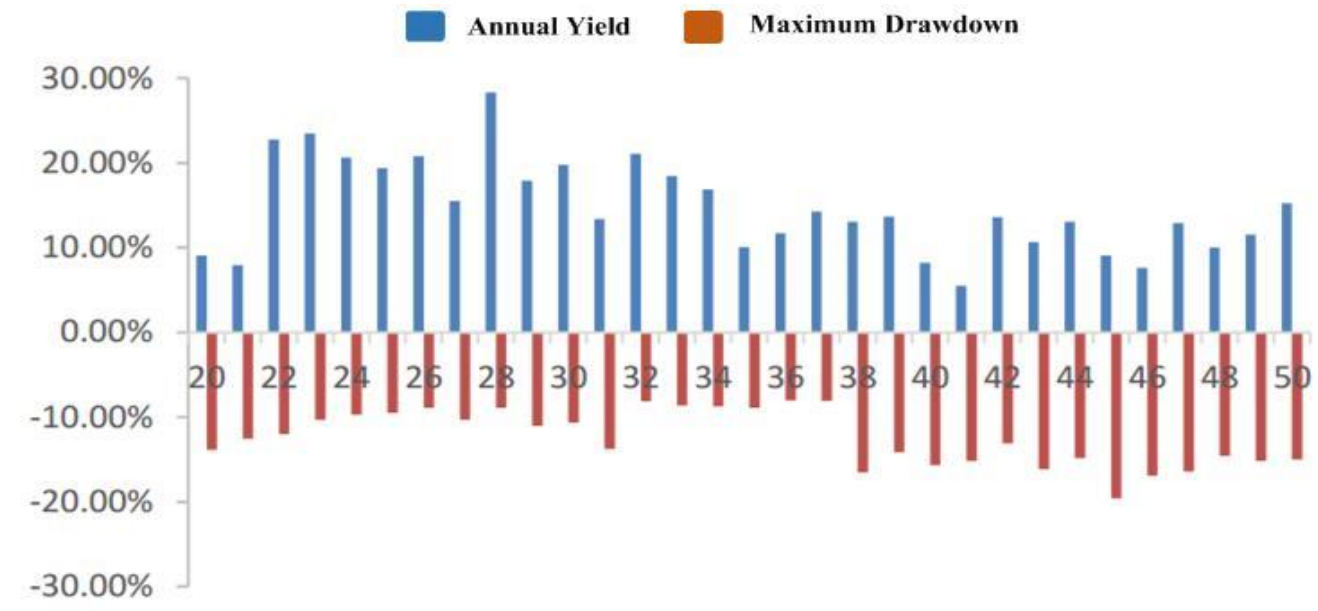

Figure 8. Performance of strategy at different warehouse opening times (out of sample) 
Table 3. Performance of strategy at different warehouse opening times (out of sample)

\begin{tabular}{cccccc}
\hline $\begin{array}{c}\text { Parameter } \\
\text { (min) }\end{array}$ & $\begin{array}{c}\text { Annualized rate of } \\
\text { return }\end{array}$ & $\begin{array}{c}\text { Maximum } \\
\text { retracement }\end{array}$ & $\begin{array}{c}\text { Parameter } \\
\text { (min) }\end{array}$ & $\begin{array}{c}\text { Annualized rate of } \\
\text { return }\end{array}$ & $\begin{array}{c}\text { Maximum } \\
\text { retracement }\end{array}$ \\
\hline $\mathbf{2 0}$ & $9.06 \%$ & $-13.84 \%$ & 36 & $11.69 \%$ & $-8.02 \%$ \\
$\mathbf{2 1}$ & $7.92 \%$ & $-12.54 \%$ & 37 & $14.25 \%$ & $-8.06 \%$ \\
$\mathbf{2 2}$ & $22.77 \%$ & $-11.98 \%$ & 38 & $13.07 \%$ & $-16.50 \%$ \\
$\mathbf{2 3}$ & $23.47 \%$ & $-10.29 \%$ & 39 & $13.64 \%$ & $-14.13 \%$ \\
$\mathbf{2 4}$ & $20.61 \%$ & $-9.71 \%$ & 40 & $8.23 \%$ & $-15.65 \%$ \\
$\mathbf{2 5}$ & $19.40 \%$ & $-9.47 \%$ & 41 & $5.51 \%$ & $-15.14 \%$ \\
$\mathbf{2 6}$ & $20.79 \%$ & $-8.89 \%$ & 42 & $13.63 \%$ & $-13.11 \%$ \\
$\mathbf{2 7}$ & $15.53 \%$ & $-10.31 \%$ & 43 & $10.68 \%$ & $-16.14 \%$ \\
$\mathbf{2 8}$ & $28.32 \%$ & $-8.87 \%$ & 44 & $9.06 \%$ & $-14.81 \%$ \\
$\mathbf{2 9}$ & $17.92 \%$ & $-11.03 \%$ & 45 & $7.59 \%$ & $-19.58 \%$ \\
$\mathbf{3 0}$ & $19.75 \%$ & $-10.66 \%$ & 46 & $12.91 \%$ & $-16.92 \%$ \\
$\mathbf{3 1}$ & $13.41 \%$ & $-13.72 \%$ & 47 & $10.03 \%$ & $-16.40 \%$ \\
$\mathbf{3 2}$ & $21.09 \%$ & $-8.15 \%$ & 48 & $11.54 \%$ & $-14.55 \%$ \\
$\mathbf{3 3}$ & $18.47 \%$ & $-8.63 \%$ & 49 & $15.23 \%$ & $-15.18 \%$ \\
$\mathbf{3 4}$ & $16.88 \%$ & $-8.71 \%$ & 50 & & $-15.00 \%$ \\
$\mathbf{3 5}$ & $10.07 \%$ & $-8.90 \%$ & & & \\
\hline
\end{tabular}

\section{Conclusion}

This study adopted the stock index futures market in early trading. A predictive model is established by using a recurrent neural network in order to evaluate the trend and shock condition of the market and forecast the probability of daily index futures market trend strategy profit. Based on this probability, the suitability of the day for trend trading is determined and whether to open a position is also decided. Empirical shows that through the trading strategy, smaller trading signal with smaller profit opportunities will be filtered while the strategy will only perform trend trading if probability of profit is high. In this way, this strategy significantly improved the profitability of the policy. In addition, the empirical results show that the strategy has good parameter stability through different transaction cost settings or different parameters of the policy retest.

\section{Reference}

Chong, T. L. (2007). A comparison of MA and RSI returns with exchange rate intervention. Applied Economics Letters, 14(5), 371-383. https://doi.org/10.1080/13504850500426269

Chi, S. C., Chen, H. P., \& Cheng, C. H. (1999). A forecasting approach for stock index future using grey theory and neural networks[C]// International Joint Conference on Neural Networks. IEEE, 1999:3850-3855, 6. 10.1109/IJCNN.1999.830769

Li, B., Zhang, D., \& Zhou, Y. (2017). There Trends in Chinese Commodity Futures Market. Securities Market Herald, (1), 43-51.

PaoloTenti. (1996) Forecasting foreign exchange rates using recurrent neural networks. Applied Artificial Intelligence, $10(6), 567-582$.

Venna, J., Kaski, S., \& Peltonen, J. (2003). Visualizations for Assessing Convergence and Mixing of MCMC[M]// Machine Learning: ECML 2003. Springer Berlin Heidelberg, 432-443.https://doi.org/10.1007/10955628_39

Yu, M. Z. (2015). Development and improvement of futures program trading system based on trading pioneer and turtle trading rules, China Market, 2015(29), 225-226.

Yan, C., \& Xuan, C. W. (2015). A Study on High-Frequency Trading Strategy Based on Variable Selection and Genetic Network. Chinese Journal of Management $\quad$ Science, $23(10)$, $47-56$. https://doi.org/10.16381/j.cnki.issn1003-207x.2015.10.006

\section{Copyrights}

Copyright for this article is retained by the author(s), with first publication rights granted to the journal.

This is an open-access article distributed under the terms and conditions of the Creative Commons Attribution license which permits unrestricted use, distribution, and reproduction in any medium, provided the original work is properly cited. 